\title{
Alzheimer's Disease Clinical Trials: Where are we now?
}

Can. J. Neurol. Sci. 2006; 33: 5

\begin{abstract}
Alzheimer's disease (AD) is becoming more frequent in Canada and all nations as the population of the world ages and more individuals survive into the risk period for this progressive and fatal brain disease. Over the course of the decade required to traverse the disorder from diagnosis to death, the patient experiences gradual decline with loss of cognition, deterioration in the ability to perform activities of daily living, and the progressive emergence of a variety of behavioral changes. Patients succumb from aspiration pneumonia, urinary tract infections, complications of decubitus ulcerations or from agerelated disorders such as stroke and cancer. There is a progressive dependency with increased caregiver burden and eventual nursing home placement of many patients. The total economic, social, and emotional tolls of AD are enormous. The disorder threatens to overwhelm social security and retirement programs for the elderly if more effective means of preventing, deferring or treating AD are not found. Currently approved symptomatic therapies including cholinesterase inhibitors (i.e., donepezil, rivistigmine, galantamine) and memantine delay deterioration by six to twelve months but do not markedly alter the course of the disease.
\end{abstract}

The single pathway from promising therapies for $\mathrm{AD}$ identified in preclinical studies to treatments available on the market is through randomized clinical trials (RCTs) comparing the active agent to a placebo in carefully diagnosed patients. Since RCTs were initially conducted for tacrine - the first cholinesterase treatment of AD - there has been a steady advance in understanding how best to conduct clinical trials to assess drugs with potential anti-AD properties. In this issue of CJNS, Feldman and colleagues ${ }^{1}$ report the proceedings of the 2 nd Canadian Conference on Antidementia Drug Guidelines held in Montreal in 2004 and produce the most comprehensive guidelines for the conduct of antidementia trials currently available. The conference included representatives from the major Canadian medical societies, members of the Central Nervous System Division of the Therapeutic Products Directorate of Health Canada, participants from the Alzheimer Society of Canada, and a variety of consultants including the Chair of the International Working Group on the Harmonization of Antidementia Drug Guidelines and a member of the Advisory Panel on Dementia of the United States Food and Drug Administration.

The principal topics addressed include description of diagnostic approaches to insure that specific inclusion criteria are met; discussion of major therapeutic approaches including trials for symptomatic agents, designs for trials of diseasemodifying agents, and designs for disease prevention in primary prevention trials of asymptomatic individuals; review of ethical aspects of RCTs; presentation of issues affecting execution of RCTs including the phases of drug development, different study designs, and approaches to statistical analysis of data generated in RCTs; discussion of the principal outcome measures currently employed in RCTs including tools to assess changes in cognition, neuropsychiatric symptoms, activities of daily living, global function, and quality of life; description of biological markers that might be integrated into RCTs (cerebrospinal fluid and blood measures, neuroimaging, and pharma-cognomics); and presentation of the major aspects of trials involving a variety of dementias and cognitive disorders including AD, mild cognitive impairment, vascular dementia, Parkinson's disease dementia, dementia with Lewy bodies, and frontotemporal dementia. Each section is followed by specific, practical recommendations for those planning, conducting, or interpreting antidementia trials. A forward-looking aspect of these guidelines is that they anticipate and discuss challenges relevant to RCTs of the evolving disease-modifying therapies as well as the already familiar trials of symptomatic agents.

The guidelines summary can address only those topics identified for discussion in the 2nd Canadian Conference on Antidementia agents, and one wishes that a few other issues that comprise barriers to current trials were included on the agenda. For example, recruitment of subjects into trials is slow, often lags behind projections, and is a major source of delay in completing RCTs. Recommendations from this group of experienced and informed individuals on how to improve trial recruitment would have been welcome. Similarly, the problem of determining the optimal dose or dose range for RCTs receives little attention in this document. Extrapolating doses from animal models that are often incomplete simulacra of AD through Phase I trials of healthy volunteers to Phases II and III of drug development is complex and worthy of discussion in an expert forum such as this. Nevertheless, the Canadian Guidelines document is the most comprehensive guidance currently available for antidementia RCTs.

Improving knowledge of the molecular biology of $\mathrm{AD}$ and other dementias has led to the identification of therapeutic targets and the emergence of compounds that are effective in animal models. These agents are promising candidates for antidementia therapies and must be tested in RCTs. The Canadican Guidelines will serve as a valuable Baedeker for the pharmaceutical industry, biotechnology companies, academics and others interested in performing RCTs of antidementia agents conducted with the highest standards.

\section{Jeffrey Cummings Los Angeles, California, USA}

1. Feldman HH, Gauthier S, Chertkow H, et al. Canadian Guidelines for the development of antidementia therapies: A conceptual summary. Can J Neurol Sci. 2006; 33: 6-26. 